
Not for reproduction, distribution or commercial use.

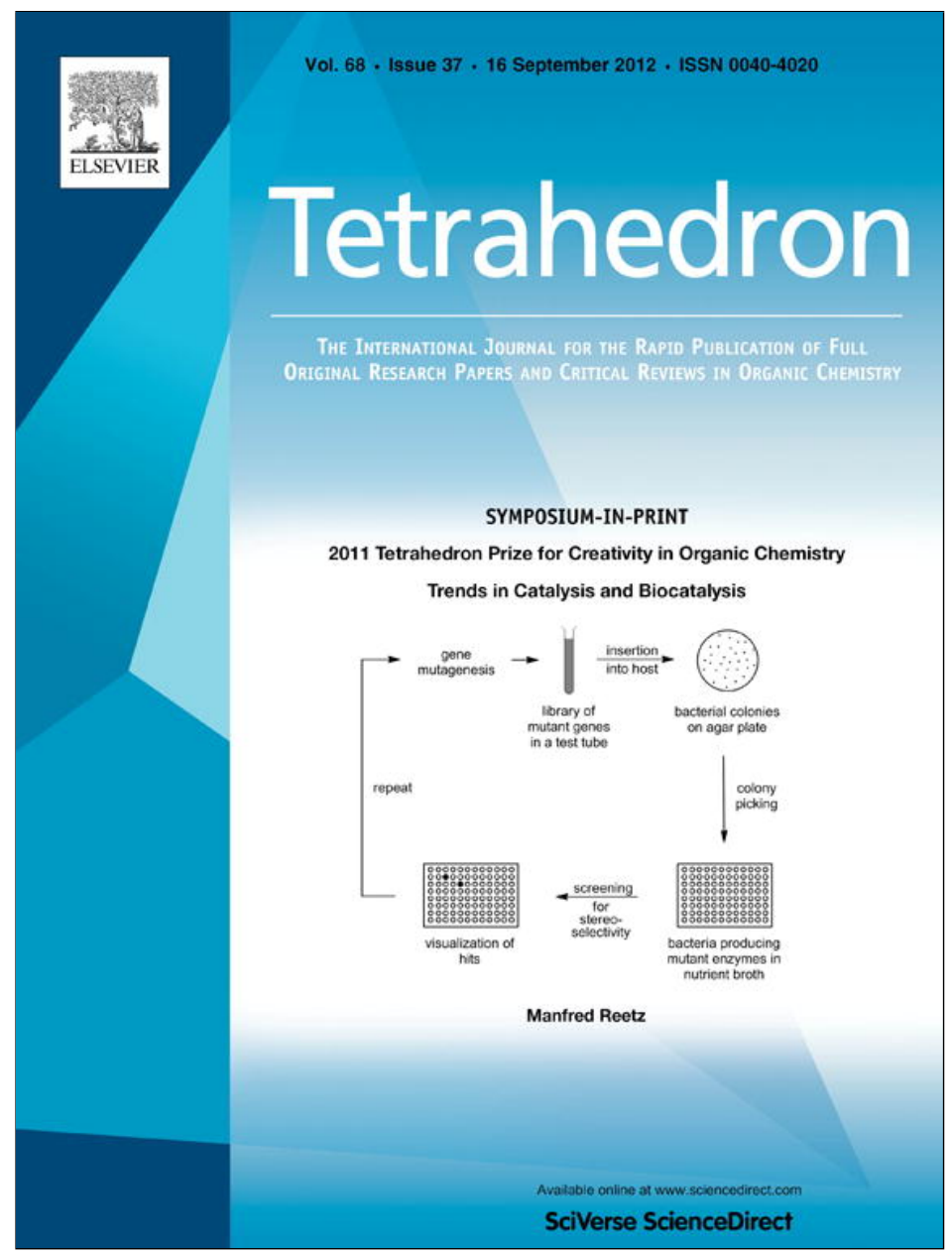

This article appeared in a journal published by Elsevier. The attached copy is furnished to the author for internal non-commercial research and education use, including for instruction at the authors institution and sharing with colleagues.

Other uses, including reproduction and distribution, or selling or licensing copies, or posting to personal, institutional or third party websites are prohibited.

In most cases authors are permitted to post their version of the article (e.g. in Word or Tex form) to their personal website or institutional repository. Authors requiring further information regarding Elsevier's archiving and manuscript policies are encouraged to visit:

http://www.elsevier.com/copyright 


\title{
Chemoenzymatic preparation of optically active trans- and cis-cyclohex-4-ene- 1,2-diamine and trans-6-aminocyclohex-3-enol derivatives
}

\author{
F. Javier Quijada, Francisca Rebolledo*, Vicente Gotor* \\ Departamento de Química Orgánica e Inorgánica, Universidad de Oviedo, 33071 Oviedo, Spain
}

\section{A R T I C L E I N F O}

\section{Article history:}

Received 29 February 2012

Received in revised form 25 April 2012

Accepted 22 May 2012

Available online 28 May 2012

\section{Keywords:}

Vicinal diamines

Aminolysis

Vicinal amino alcohols

Transesterification

Lipase

\begin{abstract}
A B S T R A C T
Lipase from Burkholderia cepacia (PSL-C) effectively catalyzed the kinetic resolution of both racemic trans- $N, N$-diallylcyclohex-4-ene-1,2-diamine $( \pm)-6$ and its precursor trans-6-(diallylamino)cyclohex-3enol $( \pm)-5$. The resulting optically active vicinal diamine and $\beta$-amino alcohol were converted into a precursor of oseltamivir and a cis-cyclohex-4-ene-1,2-diamine derivative, respectively.
\end{abstract}

(c) 2012 Elsevier Ltd. All rights reserved.

\section{Introduction}

Oseltamivir phosphate (1, Tamiflu), a carbocyclic analogue of sialic acid, is the most widely used antiviral drug for the treatment and prevention of influenzas. This compound acts by blocking the active site of neuraminidase, a glycoprotein expressed by both influenza A and B viruses. ${ }^{1}$ Between the variety of synthetic approaches to oseltamivir, ${ }^{2}$ those described by Shibasaki and Kanai et al. start from epoxide 4 , which is converted into the optically active diamine derivative $\mathbf{2}^{3}$ or into another non-symmetrical diprotected trans-cyclohex-4-ene-1,2-diamine. ${ }^{4}$ In both cases, eight steps were necessary to access to the corresponding $(1 S, 2 S)$ diamine derivative, the key step being the catalytic asymmetric ring-opening of the meso- $N$-3,5-dinitrobenzoylaziridine 3 with $\mathrm{TMSN}_{3}$ (Fig. 1).

On the other hand, we have developed in the last years an interesting chemoenzymatic method to obtain enantioenriched transcycloalkane-1,2-diamines starting from the corresponding epoxide. ${ }^{5}$ The good results attained, in both optical and chemical yields, encouraged us to apply this methodology to the synthesis of optically active trans-cyclohex-4-ene-1,2-diamine derivatives. Thus, we report herein an alternative chemoenzymatic preparation of the diamine precursor of oseltamivir. Moreover, taking into account the wide interest of $\beta$-amino alcohols and vicinal diamines in different chemical areas (medicinal, synthetic or supramolecular), ${ }^{6,7}$ we also

\footnotetext{
* Corresponding authors. Tel./fax: +34 985103448; e-mail addresses: frv@uniovi.es (F. Rebolledo),vgs@uniovi.es, vgs@fq.uniovi.es (V. Gotor).
}

have carried out the enzymatic resolution of $\beta$-amino alcohol $\mathbf{5}$, as well as its subsequent conversion into an optically active cis-cyclohex-4-ene-1,2-diamine derivative.

\section{Results and discussion}

\subsection{Chemoenzymatic preparation of optically active trans-cyclohex-4-ene-1,2-diamine derivatives}

Epoxide 4 was obtained from 1,4-cyclohexadiene as described by Albeck et al. ${ }^{8}$ Ring-opening of $\mathbf{4}$ with diallylamine yielded racemic trans-6-(diallylamino)cyclohex-3-enol $( \pm)-\mathbf{5}$, which was one-pot converted into the trans-diamine $( \pm)-6$ by the successive treatment with mesyl chloride and aqueous ammonia. In this process an aziridinium intermediate is formed as a consequence of the $\mathrm{S}_{\mathrm{N}} 2$ intramolecular displacement of the mesylate group by the vicinal tertiary amine. The subsequent attack of ammonia to the

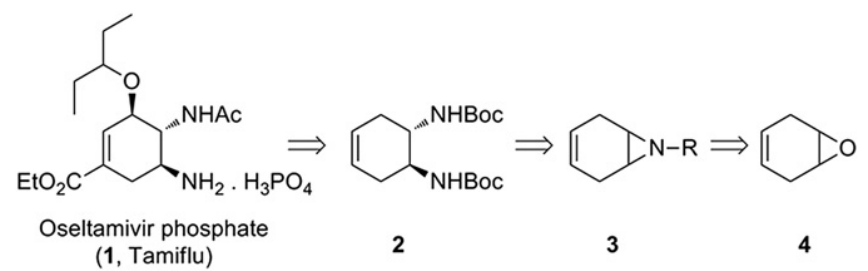

Fig. 1. Diamine derivative 2 as a precursor in the synthesis of oseltamivir (Ref. 3). 
meso-aziridinium cation ensures the trans configuration of the resulting diamine $\mathbf{6}$ (Scheme 1).<smiles>C=CCN(CC=C)[C@@H]1CC=CC[C@H]1O</smiles>

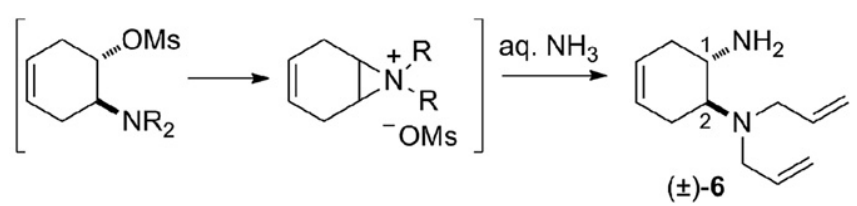

Scheme 1. Synthesis of racemic trans-diamine ( \pm )-6.

Resolution of ( \pm )-6 was accomplished by enzymatic aminolysis using ethyl acetate as the acyl donor and the solvent. At first, we tried the resolution of $( \pm)-\mathbf{6}$ using lipase B from Candida antarctica (CAL-B) as catalyst. This lipase had shown both great activity and high enantioselectivity in the resolution of racemic trans- $N, N$-diallylcyclohexane-1,2-diamine, a saturated analogue of $( \pm)-6^{5 c}$ However, its reaction with $( \pm)-\mathbf{6}$ was very slow (Table 1 , entry 1 ), the acetylation of the diamine taking place with a very low enantioselectivity $(E=3) .{ }^{9}$ Other combinations of acylating agent and solvent were assayed, but in no case was the reaction of practical utility (entries 2-4), the best result being obtained with ( \pm )-1phenylethyl acetate and tert-butyl methyl ether (TBME). ${ }^{10}$ Fortunately, a remarkable improvement was achieved when CAL-B was replaced by the lipase from Burkholderia cepacia (PSL-C). ${ }^{11}$ This lipase transformed the substrate with total enantioselectivity, the remaining diamine $(1 S, 2 S)-\mathbf{6}$ and the produced acetamide $(1 R, 2 R)$ 7 being obtained in enantiopure form after $48 \mathrm{~h}$ of reaction (Table 1 , entry 5). In this process, both compounds were isolated in very high yields. The absolute configuration $(1 S, 2 S)$ of the remaining substrate 6 was established after its transformation into (1S,2S)-2 (see Scheme 2). This means that the enzyme shows preference for the enantiomer $(1 R, 2 R)$ of the amine, thus following the Kazlauskas' rule. $^{12}$

Since the allyl groups on the tertiary nitrogen can be easily and selectively removed in the presence of other protecting groups, the method developed here could be applied to the synthesis of a variety of trans-cyclohex-4-ene-1,2-diamine derivatives in the two enantiomeric forms. As a proof, $(1 S, 2 S)-6$ was converted into $(1 S, 2 S)-2$, precursor of the oseltamivir (Scheme 2). Thus, treatment

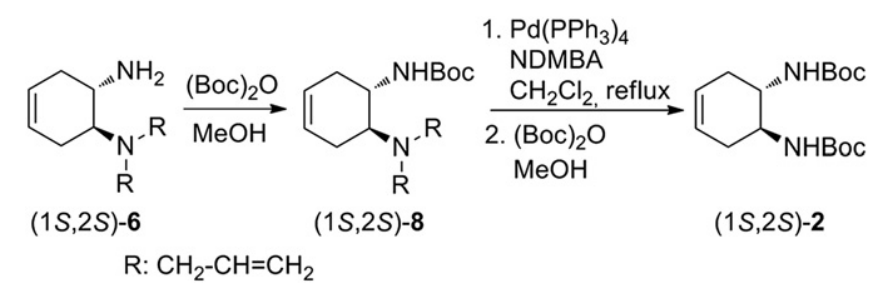

Scheme 2. Transformation of (1S,2S)-6 into $(1 S, 2 S)-\mathbf{2}$.

of $(1 S, 2 S)-\mathbf{6}$ with di-tert-butyl pyrocarbonate yielded carbamate $(1 S, 2 S)-8$, which was submitted to reaction with $N, N^{\prime}$-dimethylbarbituric acid (NDMBA) as the allyl group scavenger and $\operatorname{Pd}(0)$ as catalyst. ${ }^{13}$ In these conditions, removal of both allyl groups took place. The resulting crude mono-Boc diamine derivative was next treated with di-tert-butyl pyrocarbonate to give $(1 S, 2 S)-\mathbf{2}$ in very high yield (92\%, calculated from $\mathbf{6})$. The sign of the optical rotation of the thus obtained di-carbamate $\mathbf{2}$ matched the described value for $(1 S, 2 S)$-2 (see Experimental section).

\subsection{Enzymatic resolution of $\beta$-amino alcohol ( \pm )-5. Synthesis of an optically active cis-cyclohexen-4-ene-1,2-diamine derivative}

Enzymatic resolution of racemic trans-6-(diallylamino)cyclohex-3-enol $( \pm)-\mathbf{5}$ was carried out by transesterification reaction using PSL-C as catalyst, ${ }^{14}$ vinyl acetate as the acyl donor and TBME as the solvent (Scheme 3). Similarly to the preceding aminolysis process, the enzyme showed a great efficacy, catalyzing the acetylation of the $(1 R, 6 R)$ enantiomer of the amino alcohol $\mathbf{5}$. Thus, after $48 \mathrm{~h}$ of reaction $\left(28^{\circ} \mathrm{C}\right)$, the remaining substrate $(1 S, 6 S)-\mathbf{5}$ and the produced acetate $(1 R, 6 R)-\mathbf{9}$ were obtained with very high enantiomeric excesses and yields.

The $(1 R, 6 R)$ enantiopreference of the lipase was demonstrated by the chemical correlation of the remaining substrate $(1 S, 6 S)-\mathbf{5}$ with the configurationally known derivative (1S,2S)-12 (Scheme 4). At first, allyl groups were removed as indicated in Scheme 2 . The resulting free $(1 S, 6 S)$-6-aminocyclohex-3-enol [(1S,6S)-10] was converted into the tert-butyl carbamate $(1 S, 6 S)$-11. Catalytic hydrogenation of $\mathbf{1 1}$ yielded the tert-butyl carbamate 12. Comparison of the sign of the optical rotation of $\mathbf{1 2}$ with that published for $(1 S, 2 S)-12\left([\alpha]_{\mathrm{D}}^{20}+19.19 ; \text { c } 0.50, \mathrm{MeOH}\right)^{15}$ establishes the $(1 S, 2 S)$ configuration for the remaining substrate of the enzymatic transesterification.

Moreover, in order to show the synthetic utility of the enzymatically prepared compounds, the optically active cis-diamine derivative (1S,2R)-15 was prepared from the tert-butyl carbamate

Table 1

Lipase-catalyzed resolution of diamine $( \pm)-\mathbf{6}$<smiles>C=CCN(CC=C)[C@H]1CC=CC[C@H]1N</smiles>

$( \pm)-6$

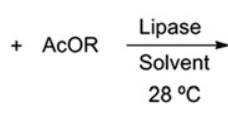

$28^{\circ} \mathrm{C}$

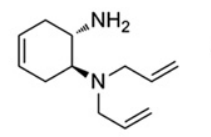

$(1 S, 2 S)-6$

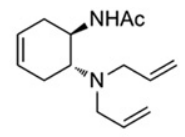

$(1 R, 2 R)-7$

\begin{tabular}{|c|c|c|c|c|c|c|c|c|}
\hline Entry & Enzyme & Acyl donor (AcOR) & Solvent & Time (days) & Remaining substrate $(1 S, 2 S)-\mathbf{6}$, ee $(\%)$ & Product $(1 R, 2 R)-\mathbf{7}$ eе $\mathrm{P}(\%)$ & $c^{\mathrm{a}}(\%)$ & $E^{\mathrm{b}}$ \\
\hline 1 & CAL-B & AcOEt & AcOEt & 5 & 11 & 41 & 21 & 3 \\
\hline 2 & CAL-B & $\mathrm{AcOEt}^{\mathrm{c}}$ & TBME & 7 & 4 & 85 & 4 & 12 \\
\hline 3 & CAL-B & $\mathrm{AcOCH}(\mathrm{Ph}) \mathrm{CH}_{3}{ }^{\mathrm{d}}$ & TBME & 5 & 12 & 93 & 11 & 31 \\
\hline 4 & CAL-B & $\mathrm{AcOCH}(\mathrm{Ph}) \mathrm{CH}_{3}{ }^{\mathrm{d}}$ & THF & 7 & 6 & 83 & 7 & 11 \\
\hline 5 & PSL-C & AcOEt & AcOEt & 2 & $>99^{\mathrm{e}}$ & $>99^{\mathrm{e}}$ & 50 & $>200$ \\
\hline
\end{tabular}

a Conversion degree: $c=\mathrm{ee}_{\mathrm{s}} /\left(\mathrm{ee}_{\mathrm{s}}+\mathrm{ee}_{\mathrm{p}}\right)$.

b Determined from ees and ee $e_{\mathrm{p}}$ as in Ref. 9.

c A molar ratio ester/diamine of 6:1 was used.

d A molar ratio ester/diamine of 3:1 was used.

e Isolated yields after flash chromatography were $42 \%$ and $47 \%$ for $(1 S, 2 S)-\mathbf{6}$ and $(1 R, 2 R)-\mathbf{7}$, respectively. 
<smiles>C=CCN(CC=C)C1CC=CCC1O</smiles>
$\mathrm{AcOCH}=\mathrm{CH}_{2}$
$\stackrel{\mathrm{PSL}-\mathrm{C}}{\longrightarrow}$
$\underset{2 B M E}{28^{\circ} \mathrm{C}}$
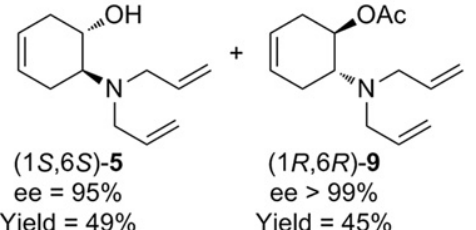

Scheme 3. Enzymatic resolution of $( \pm)-\mathbf{5}$.

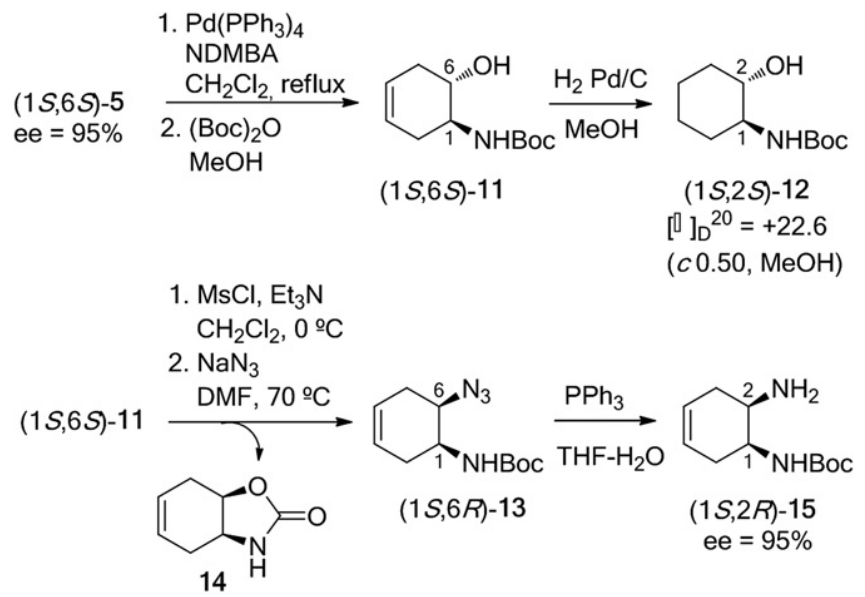

Scheme 4. Assignment of the absolute configuration of the enzymatically prepared $\mathbf{5}$ and its transformation into the cis-diamine $(1 S, 2 R)-\mathbf{1 5}$

$(1 S, 6 S)$-11. Thus, treatment of $\mathbf{1 1}$ with mesyl chloride gave the mesylate derivative, which was subsequently treated with sodium azide in DMF at $70{ }^{\circ} \mathrm{C}$. In this reaction, beside the cis-azido carbamate $(1 S, 6 R)-\mathbf{1 3}$, the oxazolidinone $\mathbf{1 4}$ was also isolated as a minor product (see experimental section). This bicyclic compound is the result of an intramolecular $\mathrm{S}_{\mathrm{N}} 2$ displacement of the mesylate group by the carbonyl oxygen of the Boc group. ${ }^{16}$ To minimize the formation of 14, the reaction with sodium azide was also tried at a lower temperature $\left(50{ }^{\circ} \mathrm{C}\right)$ but under these reaction conditions, compound 13 was not formed. Finally, the Staudinger reduction of the azide function of $(1 S, 6 R)$-13 yielded the cis-amino carbamate $(1 S, 2 R)-15$. In all the synthetic approaches no racemization took place as confirmed by chiral-HPLC analysis of $\mathbf{1 5}$.

\section{Conclusions}

Efficient chemoenzymatic approaches to optically active transcycohex-4-ene-1,2-diamine and trans-6-aminocyclohex-3-enol derivatives have been developed, in both cases the key step being the kinetic resolution of the corresponding racemic compound catalyzed by PSL-C. Moreover, the preparation of a precursor of oseltamivir as well as an optically active cis-cyclohex-4-ene-1,2diamine derivative have also been carried out.

\section{Experimental section}

\subsection{General}

Lipase B from C. antarctica (Novozyme 435, available immobilized on polyacrylamide, $7300 \mathrm{PLU} / \mathrm{g}$ ) was gifted by Novo Nordisk Co. Immobilized lipase from Burkholderia cepacia (PSL-C, $783 \mathrm{U} / \mathrm{g}$ ) was purchased from Amano Pharmaceutical Co. For the enzymatic reactions, ethyl acetate of spectrophotometric grade (stored with $4 \AA$ molecular sieves), anhydrous TBME or THF and ( \pm )-1phenylethyl acetate were used. Melting points were taken on samples in open capillary tubes and are uncorrected. ${ }^{1} \mathrm{H}$ NMR and proton-decoupled ${ }^{13} \mathrm{C}$ NMR spectra $\left(\mathrm{CDCl}_{3}\right.$ solutions) were obtained using AC-300 or DPX-300 $\left({ }^{1} \mathrm{H}, 300.13 \mathrm{MHz}\right.$ and ${ }^{13} \mathrm{C}$, $75.5 \mathrm{MHz})$ and $\mathrm{AV}-400 \mathrm{MHz}\left({ }^{1} \mathrm{H}, 400.13 \mathrm{MHz}\right.$ and $\left.{ }^{13} \mathrm{C}, 100.63 \mathrm{MHz}\right)$ spectrometers using the $\delta$ scale (parts per million) for chemical shifts; calibration was made on the $\mathrm{CDCl}_{3}\left({ }^{13} \mathrm{C} ; 76.95 \mathrm{ppm}\right)$ or the residual $\mathrm{CHCl}_{3}\left({ }^{1} \mathrm{H} ; 7.26 \mathrm{ppm}\right)$ signals.

\subsection{Procedures and characterization of products}

4.2.1. ( \pm )-trans-6-(Diallylamino)cyclohex-3-en-1-ol [( \pm )-5].<smiles>C=CCN(C1CC=CCC1O)C(C)(C)/C=C\C</smiles>

Diallylamine (10.3 mL, $84.0 \mathrm{mmol})$ was added to a solution of epoxide 4 (3.80 g, $40.0 \mathrm{mmol})$ in deoxygenated ethanol $(80 \mathrm{~mL})$. After refluxing under a nitrogen atmosphere for $48 \mathrm{~h}$, the solvent and the excess amine were evaporated in vacuo to give the crude product, which was purified by distillation to reduced pressure to give the title compound $( \pm)-\mathbf{5}\left(\mathrm{bp}=54{ }^{\circ} \mathrm{C}, 0.01 \mathrm{Torr} ; 5.00 \mathrm{~g}, 66 \%\right)$ as a colourless oil; [found: $\mathrm{C}, 74.8 ; \mathrm{H}, 10.2 ; \mathrm{N}$ 7.5. $\mathrm{C}_{12} \mathrm{H}_{19} \mathrm{NO}$ requires $\mathrm{C}$, $74.57 ; \mathrm{H}, 9.91 ; \mathrm{N}, 7.25 \%] ; R_{f}$ (hexane/AcOEt 10:1) $0.21 ; \nu_{\max }\left(\mathrm{CH}_{2} \mathrm{Cl}_{2}\right)$ $3412,1630,1527,1120 \mathrm{~cm}^{-1} ; \delta_{\mathrm{H}}\left(400 \mathrm{MHz} \mathrm{CDC1}_{3}\right) 5.80$ (dddd, $2 \mathrm{H}$, $\left.{ }^{3} J_{C, A} 4.4 \mathrm{~Hz},{ }^{3} J_{C, B} 8.0 \mathrm{~Hz},{ }^{3} J_{C, D} 10.2 \mathrm{~Hz},{ }^{3} J_{C, E} 14.9 \mathrm{~Hz}, \mathrm{H}_{\mathrm{C}}\right), 5.63-5.50(\mathrm{~m}$, $2 \mathrm{H}, \mathrm{H}-3$ and $\mathrm{H}-4), 5.22-5.08\left(\mathrm{~m}, 4 \mathrm{H}, \mathrm{H}_{\mathrm{D}}\right.$ and $\mathrm{H}_{\mathrm{E}}$ ), 3.69 (ddd, $1 \mathrm{H},{ }^{3} \mathrm{~J} 6.1$, 9.5, $10.8 \mathrm{~Hz}, \mathrm{H}-1$ ), 3.33 (ddt, $2 \mathrm{H},{ }^{4} J_{\mathrm{A}, \mathrm{D}}={ }^{4} J_{\mathrm{A}, \mathrm{E}}=1.7 \mathrm{~Hz},{ }^{3} J_{\mathrm{A}, \mathrm{C}} 4.4 \mathrm{~Hz},{ }^{2} J_{\mathrm{A}, \mathrm{B}} \mid$ $\left.14.2 \mathrm{~Hz}, \mathrm{H}_{\mathrm{A}}\right), 2.95\left(\mathrm{dd}, 2 \mathrm{H},{ }^{3} J_{\mathrm{B}, \mathrm{C}} 8.0 \mathrm{~Hz},\left.\right|^{2} J_{\mathrm{B}, \mathrm{A}} \mid 14.2 \mathrm{~Hz}, \mathrm{H}_{\mathrm{B}}\right), 2.83(\mathrm{dt}, 1 \mathrm{H}$, $3 \mathrm{~J} 5.6$ (d), 10.8 (t) Hz, H-6), 2.58-2.50 (m, 1H), 2.21-2.12 (m, 1H), 2.09-1.97 (m, 2H); $\delta_{\mathrm{C}}\left(75.5 \mathrm{MHz} \mathrm{CDC}_{3}\right) 136.3\left(\mathrm{CH}=\mathrm{CH}_{2}\right), 125.1(\mathrm{C}-3$ or C-4), $124.5(\mathrm{C}-4$ or $\mathrm{C}-3), 117.3\left(\mathrm{CH}=\mathrm{CH}_{2}\right), 65.7(\mathrm{CH}), 61.1(\mathrm{CH}), 52.4$ $\left(\mathrm{N}-\mathrm{CH}_{2}\right), 33.8\left(\mathrm{CH}_{2}\right), 22.7\left(\mathrm{CH}_{2}\right) ; \mathrm{m} / z\left(\mathrm{ESI}^{+}\right) 194\left(100, \mathrm{MH}^{+}\right)$.

4.2.2. ( \pm )-trans-N,N-Diallylcyclohex-4-ene-1,2-diamine $[( \pm)-6]$. To a cooled $\left(0^{\circ} \mathrm{C}\right)$ solution of $( \pm)-5(1.50 \mathrm{~g}, 7.70 \mathrm{mmol})$ in anhydrous diethyl ether $(17 \mathrm{~mL})$, triethylamine $(1.7 \mathrm{~mL}, 12.4 \mathrm{mmol})$ and mesyl chloride $(0.720 \mathrm{~mL}, 9.30 \mathrm{mmol})$ were added. The stirred mixture was allowed to warm to room temperature and, after $30 \mathrm{~min}$, triethylamine $(2.2 \mathrm{~mL}, 15.5 \mathrm{mmol})$ and concentrated aqueous $\mathrm{NH}_{3}$ $(20 \mathrm{~mL})$ were added. The resulting two-phase reaction mixture was vigorously stirred for $12 \mathrm{~h}$. The layers were separated, and the aqueous layer was extracted with diethyl ether $(420 \mathrm{~mL})$. All the organic layers were combined and successively washed with aqueous $3 \mathrm{M} \mathrm{NaOH}(20 \mathrm{~mL})$ and brine $(20 \mathrm{~mL})$. After removal of the organic solvents under reduced pressure, the obtained crude product was purified by flash chromatography (a gradient of AcOEt to AcOEt/methanol 4:1 was used as eluent) to give the title compound ( \pm )-6 (78\%) as a colourless oil; [found: C, 74.8; H, 10.7; N 14.3. $\mathrm{C}_{12} \mathrm{H}_{20} \mathrm{~N}_{2}$ requires $\mathrm{C}, 74.95 ; \mathrm{H}, 10.48 ; \mathrm{N}, 14.57 \%$ ]; $R_{f}$ (hexane/ AcOEt 1:1) $0.17 ; \nu_{\max }\left(\mathrm{CH}_{2} \mathrm{Cl}_{2}\right) 3420,3347,2980,1634 \mathrm{~cm}^{-1} ; \delta_{\mathrm{H}}$ (300 MHz CDC1 13 ) 5.81 (dddd, $2 \mathrm{H},{ }^{3} \mathrm{~J} 4.5,7.8,10.2,14.7 \mathrm{~Hz}, \mathrm{CH}=\mathrm{CH}_{2}$ ), 5.67-5.57 $(\mathrm{m}, 2 \mathrm{H}, \quad \mathrm{CH}=\mathrm{CH}), \quad 5.22-5.03\left(\mathrm{~m}, \quad 4 \mathrm{H}, \quad \mathrm{CH}=\mathrm{CH}_{2}\right)$, $3.34-3.20(\mathrm{~m}, 2 \mathrm{H}, \mathrm{N}-\mathrm{CHH}), 2.99-2.85(\mathrm{~m}, 3 \mathrm{H}, \mathrm{N}-\mathrm{CH} H+\mathrm{H}-1), 2.64$ [dt, $1 \mathrm{H},{ }^{3} \mathrm{~J} 5.4(\mathrm{~d}), 10.8(\mathrm{t}) \mathrm{Hz}, \mathrm{H}-2$ ], 2.50-2.38 (m, 1H), 2.20-2.08 (m, $1 \mathrm{H}), 2.19-1.77$ [m+s, $4 \mathrm{H}$. Singlet corresponds to $\left.\mathrm{NH}_{2}(1.80 \mathrm{ppm})\right]$; $\delta_{\mathrm{C}}\left(75.5 \mathrm{MHz} \mathrm{CDC}_{3}\right) 136.8\left(\mathrm{CH}=\mathrm{CH}_{2}\right), 125.2(\mathrm{CH}=\mathrm{CH}), 124.5(\mathrm{CH}=$ $\mathrm{CH}), 115.9\left(\mathrm{CH}=\mathrm{CH}_{2}\right), 60.8(\mathrm{CH}), 52.0\left(\mathrm{~N}-\mathrm{CH}_{2}\right), 47.6(\mathrm{CH}), 35.0$ $\left(\mathrm{CH}_{2}\right), 22.3\left(\mathrm{CH}_{2}\right) ; \mathrm{m} / z\left(\mathrm{ESI}^{+}\right) 193\left(100, \mathrm{MH}^{+}\right)$.

4.2.3. Enzymatic kinetic resolution of amino alcohol ( \pm )-5. To a mixture of $( \pm)-5(1.00 \mathrm{~g}, 5.10 \mathrm{mmol})$, PSL-C $(520 \mathrm{mg})$ and $4 \AA$ 
molecular sieves (50 mg), under a nitrogen atmosphere, anhydrous tert-butyl methyl ether $(30 \mathrm{~mL})$ and vinyl acetate $(1.40 \mathrm{~mL}$, $15.6 \mathrm{mmol}$ ) were added. The mixture was stirred for $48 \mathrm{~h}$ at $28{ }^{\circ} \mathrm{C}$ and $200 \mathrm{rpm}$. After this time, the enzyme was filtered through a pad of Celite ${ }^{\circledR}$ and the solid was washed with methanol. The solution was concentrated to reduced pressure and the resulting crude, which was formed by the optically active $(1 S, 6 S)-\mathbf{5}$ and the ester $(1 R, 6 R)-\mathbf{9}$, was submitted to flash chromatography (hexane/AcOEt 30:1).

4.2.3.1. (1S,6S)-6-(Diallylamino)cyclohex-3-en-1-ol ] [(1S,6S)5]. Yield 49\%; $[\alpha]_{\mathrm{D}}^{20}+43.5\left(\right.$ c $\left.1.0, \mathrm{CHCl}_{3}\right)$, ee $95 \%$.

4.2.3.2. (1R,6R)-6-(Diallylamino)cyclohex-3-enyl acetate [(1R,6R)9]. Colourless oil; yield 45\%; [found: $\mathrm{C}, 71.7 ; \mathrm{H}, 8.7 ; \mathrm{N} \mathrm{6.2.} \mathrm{C}_{14} \mathrm{H}_{21} \mathrm{NO}_{2}$ requires $\mathrm{C}, 71.46 ; \mathrm{H}, 8.99$; $\mathrm{N}, 5.95 \%] ; R_{f}$ (hexane/AcOEt 10:1) 0.42; $[\alpha]_{\mathrm{D}}^{20}-2.6\left(\mathrm{c} 1.0, \mathrm{CHCl}_{3}\right)$, ee $>99 \% ; \nu_{\max }\left(\mathrm{CH}_{2} \mathrm{Cl}_{2}\right) 1728,1634,1503$, $1210 \mathrm{~cm}^{-1} ; \delta_{\mathrm{H}}\left(300 \mathrm{MHz} \mathrm{CDC1}_{3}\right) 5.72$ (dddd, $2 \mathrm{H}, 3 \mathrm{~J}$ 5.4, 6.9, 10.3, $\left.12.0 \mathrm{~Hz}, \mathrm{CH}=\mathrm{CH}_{2}\right), 5.61-5.55(\mathrm{~m}, 1 \mathrm{H}, \mathrm{CH}=\mathrm{CH}), 5.47-5.42(\mathrm{~m}, 1 \mathrm{H}$, $\mathrm{CH}=\mathrm{CH}), 5.15[\mathrm{dq}, 2 \mathrm{H}, J 1.7$ (q), $12.0(\mathrm{~d}) \mathrm{Hz}, \mathrm{CH}=\mathrm{CHH}], 5.20-5.00(\mathrm{~m}$, $1 \mathrm{H}, \mathrm{H}-1$ ), 5.05 [dq, 2H,J 1.7 (q), 10.3 (d) Hz, CH=CHH], 3.22 (ddt, 2H, $\left.{ }^{4} \mathrm{~J} 1.4(\mathrm{t}) \mathrm{Hz},{ }^{3} \mathrm{~J} 5.0 \mathrm{~Hz},\left.\right|^{2} \mathrm{~J} \mid 14.4 \mathrm{~Hz}, \mathrm{~N}-\mathrm{CHH}\right), 3.10-2.94(\mathrm{~m}, 3 \mathrm{H}$, $\mathrm{N}-\mathrm{CH}+\mathrm{H}-6), 2.52-2.42(\mathrm{~m}, 1 \mathrm{H}), 2.24-2.02[\mathrm{~m}+\mathrm{s}, 6 \mathrm{H}$. Singlet corresponds to $\left.\mathrm{CH}_{3}(2.07 \mathrm{ppm})\right] ; \delta_{\mathrm{C}}\left(75.5 \mathrm{MHz} \mathrm{CDC}_{3}\right) 170.2(\mathrm{C}=\mathrm{O})$, $137.4\left(\mathrm{CH}=\mathrm{CH}_{2}\right), 125.8(\mathrm{CH}=\mathrm{CH}), 123.4(\mathrm{CH}=\mathrm{CH}), 115.9\left(\mathrm{CH}=\mathrm{CH}_{2}\right)$, 69.8 $(\mathrm{CH}), 57.6(\mathrm{CH}), 52.8\left(\mathrm{~N}-\mathrm{CH}_{2}\right), 31.9\left(\mathrm{CH}_{2}\right), 25.8\left(\mathrm{CH}_{2}\right), 21.2\left(\mathrm{CH}_{3}\right)$; $\mathrm{m} / \mathrm{z}\left(\mathrm{EI}^{+}\right): 235\left(15, \mathrm{M}^{\cdot+}\right), 194\left[18\left(\mathrm{M}-\mathrm{CH}_{2}-\mathrm{CH}=\mathrm{CH}_{2}\right)^{+}\right], 149$ (100).

4.2.4. Enzymatic kinetic resolution of diamine ( \pm )-6. Ethyl acetate $(24 \mathrm{~mL})$ was added to a mixture of racemic diamine $( \pm)-\mathbf{6}(0.750 \mathrm{~g}$, $3.90 \mathrm{mmol})$ and PSL-C $(0.390 \mathrm{~g})$ under a nitrogen atmosphere. The new mixture was stirred at $28^{\circ} \mathrm{C}$ and $200 \mathrm{rpm}$ during $48 \mathrm{~h}$. Then, the reaction mixture was filtered through a pad of Celite ${ }^{\circledR}$ and the solid was washed with methanol. Organic solvents were evaporated under reduced pressure and the resulting crude, which consisted of a mixture of diamine (1S,2S)-6 and monoamide $(1 R, 2 R)-\mathbf{7}$, was subjected to flash chromatogarphy (a gradient of hexane/ AcOEt 1:1 to AcOEt/methanol 4:1 was used as eluent).

4.2.4.1. (1S,2S)-N,N-Diallylcyclohex-4-ene-1,2-diamine [(1S,2S)6]. Yield $42 \%$; $[\alpha]_{\mathrm{D}}^{20}+67.3\left(\right.$ c $\left.1.0, \mathrm{CHCl}_{3}\right)$, ee $>99 \%$.

4.2.4.2. (1R,2R)-N-Acetyl- $N^{\prime}, N^{\prime}$-diallylcyclohex-4-ene-1,2diamine [(1R,2R)-7]. Yield 47\%; mp 98-100 ${ }^{\circ} \mathrm{C}$; [found: $\mathrm{C}, 71.9 ; \mathrm{H}$, 9.6; $\mathrm{N}$ 12.2. $\mathrm{C}_{14} \mathrm{H}_{22} \mathrm{~N}_{2} \mathrm{O}$ requires $\left.\mathrm{C}, 71.76 ; \mathrm{H}, 9.46 ; \mathrm{N}, 11.95 \%\right] ; R_{f}$ (hexane/AcOEt $5: 1$ ) $0.41 ;[\alpha]_{\mathrm{D}}^{20}-5.4$ (c 1.0, $\mathrm{CHCl}_{3}$ ), ee >99\%; $\nu_{\max }\left(\mathrm{CH}_{2} \mathrm{Cl}_{2}\right) 3261,3095,1634,1579 \mathrm{~cm}^{-1} ; \delta_{\mathrm{H}}\left(300 \mathrm{MHz} \mathrm{CDC}_{3}\right) 6.39$ (br s, $1 \mathrm{H}, \mathrm{NH}$ ), 5.72 (dddd, $2 \mathrm{H},{ }^{3} \mathrm{~J} 4.6,8.0,10.2,12.6 \mathrm{~Hz}, \mathrm{CH}=\mathrm{CH}_{2}$ ), 5.64-5.48 $(\mathrm{m}, 2 \mathrm{H}, \mathrm{CH}=\mathrm{CH}), 5.21-5.05\left(\mathrm{~m}, 4 \mathrm{H}, \mathrm{CH}=\mathrm{CH}_{2}\right), 3.79-3.65$ $(\mathrm{m}, 1 \mathrm{H}, \mathrm{H}-1), 3.31-3.19(\mathrm{~m}, 2 \mathrm{H}, \mathrm{N}-\mathrm{CHH}), 3.02-2.80(\mathrm{~m}, 4 \mathrm{H}$, $\mathrm{N}-\mathrm{CHH}+\mathrm{H}-2+\mathrm{CHH}), 2.28-2.12(\mathrm{~m}, 1 \mathrm{H}, \mathrm{CHH}), 2.12-1.77[\mathrm{~m}+\mathrm{s}, 5 \mathrm{H}$. Singlet corresponds to $\left.\mathrm{CH}_{3}(1.95 \mathrm{ppm})\right]$; $\delta_{\mathrm{C}}\left(75.5 \mathrm{MHz} \mathrm{CDC}_{3}\right) 170.4$ $(\mathrm{C}=\mathrm{O}), 136.5\left(\mathrm{CH}=\mathrm{CH}_{2}\right), 125.1(\mathrm{CH}=\mathrm{CH}), 124.9(\mathrm{CH}=\mathrm{CH}), 116.8$ $\left(\mathrm{CH}=\mathrm{CH}_{2}\right), 57.5(\mathrm{CH}), 52.0\left(\mathrm{~N}-\mathrm{CH}_{2}\right), 47.8(\mathrm{CH}), 33.3\left(\mathrm{CH}_{2}\right), 23.4\left(\mathrm{CH}_{3}\right)$, $23.0\left(\mathrm{CH}_{2}\right) ; \mathrm{m} / z\left(\mathrm{EI}^{+}\right)$: $193\left[70,\left(\mathrm{M}-\mathrm{CH}_{2}-\mathrm{CH}=\mathrm{CH}_{2}\right)^{+}\right], 162(100)$.

4.2.5. (1S,2S)-N-(tert-Butoxycarbonyl)- $N^{\prime}, N^{\prime}$-diallylcyclohex-4-ene1,2-diamine [(1S,2S)-8]. Di-tert-butyl pyrocarbonate $(0.419 \mathrm{~g}$, $1.90 \mathrm{mmol})$ was added to a solution of $(1 S, 2 S)-6(0.336 \mathrm{~g}$, $1.75 \mathrm{mmol})$ in methanol $(10 \mathrm{~mL})$. After $1 \mathrm{~h}$ of reaction at room temperature, solvents were evaporated and the residue was purified by flash chromatography (hexane/AcOEt $15: 1$ as eluant) to yield the title compound $(1 S, 2 S)-8(98 \%)$ as a white solid; mp 45-46 ${ }^{\circ} \mathrm{C} ; R_{f}$ (hexane/AcOEt 5:1) 0.52; $[\alpha]_{\mathrm{D}}^{20}+49.7\left(c 1.0, \mathrm{CHCl}_{3}\right)$, ee $98 \% ; \nu_{\max }\left(\mathrm{CH}_{2} \mathrm{Cl}_{2}\right)$ 3381, 2977, 1715, $1648 \mathrm{~cm}^{-1} ; \delta_{\mathrm{H}}(300 \mathrm{MHz}$ $\mathrm{CDC}_{3}$ ) 5.77 (dddd, $2 \mathrm{H},{ }^{3} \mathrm{~J} 4.8,8.0,10.2,12.7 \mathrm{~Hz}, \mathrm{CH}=\mathrm{CH}_{2}$ ), $5.62-5.50(\mathrm{~m}, 2 \mathrm{H}, \mathrm{CH}=\mathrm{CH}), 5.42(\mathrm{br} \mathrm{s}, 1 \mathrm{H}, \mathrm{NH}), 5.20-5.04(\mathrm{~m}, 4 \mathrm{H}$,
$\left.\mathrm{CH}=\mathrm{CH}_{2}\right), 3.57-3.43(\mathrm{~m}, 1 \mathrm{H}, \mathrm{H}-1), 3.32-3.18(\mathrm{~m}, 2 \mathrm{H}, \mathrm{N}-\mathrm{CH})$, 2.91-2.76 (m, 4H, N-CHH+H-2+CHH), 2.26-2.12 (m, $1 \mathrm{H}, \mathrm{CH})$, $2.08-1.83(\mathrm{~m}, 2 \mathrm{H}, 2 \times \mathrm{CHH}), 1.44\left(\mathrm{~s}, 9 \mathrm{H},{ }^{t} \mathrm{Bu}\right) ; \delta_{\mathrm{C}}\left(75.5 \mathrm{MHz} \mathrm{CDC1}_{3}\right)$ $156.0(\mathrm{C}=\mathrm{O}), 136.6\left(\mathrm{CH}=\mathrm{CH}_{2}\right), 124.9(\mathrm{CH}=\mathrm{CH}), 124.8(\mathrm{CH}=\mathrm{CH})$, 116.6 $\left(\mathrm{CH}=\mathrm{CH}_{2}\right), 78.5(\mathrm{C}), 57.6(\mathrm{CH}), 52.0\left(\mathrm{~N}-\mathrm{CH}_{2}\right), 48.3(\mathrm{CH}), 33.8$ $\left(\mathrm{CH}_{2}\right), 28.3\left(\mathrm{CH}_{3}\right), 23.0\left(\mathrm{CH}_{2}\right), \mathrm{m} / \mathrm{z}\left(\mathrm{ESI}^{+}\right) 293\left(50, \mathrm{MH}^{+}\right), 237(100)$; HRMS $\left(\mathrm{ESI}^{+}\right): \mathrm{MH}^{+}$, found 293.2230. $\mathrm{C}_{17} \mathrm{H}_{29} \mathrm{~N}_{2} \mathrm{O}_{2}$ requires 293.2224 .

4.2.6. (1S,2S)-N,N'-Di(tert-butoxycarbonyl)cyclohex-4-ene-1,2diamine $[(1 S, 2 S)-2]$. Obtained from $(1 S, 2 S)-8(0.58 \mathrm{mmol})$. The allyl groups were removed following the method described by an analogous $\mathrm{N}, \mathrm{N}$-diallylcyclopentane-1,2-diamine. ${ }^{17}$ The resulting crude mono-Boc diamine was subsequently dissolved in methanol $(4 \mathrm{~mL})$ and treated with di-tert-butyl pyrocarbonate $(0.140 \mathrm{~g}$, $0.64 \mathrm{mmol}$ ). After $5 \mathrm{~h}$ of reaction at room temperature, solvents were eliminated and the residue was subjected to flash chromatography (hexane/Et ${ }_{2} \mathrm{O} 7: 3$ as eluent) to yield the title compound $(1 S, 2 S)-2$ (94\%). Spectroscopic data are in good agreement with those previously reported. ${ }^{3}[\alpha]_{\mathrm{D}}^{20}-30.1\left(c\right.$ 1.0, $\left.\mathrm{CHCl}_{3}\right)$. Ref. 3: $[\alpha]_{\mathrm{D}}^{21}$ -34.5 (c $1.100, \mathrm{CHCl}_{3}$ ), ee $99 \%$.

4.2.7. (1S,6S)-6-Aminocyclohex-3-en-1-ol [(1S,6S)-10]. It was obtained from $(1 S, 6 S)-5(0.400 \mathrm{~g}, 2.07 \mathrm{mmol})$ following the method described for the removal of allyl groups. ${ }^{17}$ White solid; yield $82 \%$; mp 106-107 ${ }^{\circ} \mathrm{C} ; R_{f}$ (methanol) 0.18; $[\alpha]_{\mathrm{D}}^{20}+141.9\left(c 1.0, \mathrm{CHCl}_{3}\right)$, ee $95 \% ; \nu_{\max }\left(\mathrm{CH}_{2} \mathrm{Cl}_{2}\right) 3410,3368,3320,1693,1535 \mathrm{~cm}^{-1} ; \delta_{\mathrm{H}}(400 \mathrm{MHz}$ $\left.\mathrm{CDC}_{3}\right)$ 5.58-5.48 (m, 2H, CH=CH), $3.47(\mathrm{dt}, 1 \mathrm{H}, J 5.9(\mathrm{~d}), 9.6(\mathrm{t}) \mathrm{Hz}$, $\mathrm{H}-1), 2.77$ (q, 1H, $\left.{ }^{3} \mathrm{~J} 9.6 \mathrm{~Hz}, \mathrm{H}-6\right), 2.43-2.18(\mathrm{~m}, 5 \mathrm{H}, 2 \times$ $\left.\mathrm{CH}+\mathrm{OH}+\mathrm{NH}_{2}\right), 2.09-1.97(\mathrm{~m}, 1 \mathrm{H}, \mathrm{CH} H), 1.93-1.81(\mathrm{~m}, 1 \mathrm{H}, \mathrm{CH})$; $\delta_{\mathrm{C}}\left(100 \mathrm{MHz} \mathrm{CDC}_{3}\right) 125.0(\mathrm{CH}=\mathrm{CH}), 71.9(\mathrm{CH}), 53.0(\mathrm{CH}), 34.9$ $\left(\mathrm{CH}_{2}\right), 33.6\left(\mathrm{CH}_{2}\right) ; \mathrm{m} / z\left(\mathrm{ESI}^{+}\right) 114\left(100, \mathrm{MH}^{+}\right)$; $\mathrm{HRMS}\left(\mathrm{ESI}^{+}\right): \mathrm{MH}^{+}$, found 114.0919. $\mathrm{C}_{6} \mathrm{H}_{12} \mathrm{NO}$ requires 114.0913.

4.2.8. tert-Butyl (1S,6S)-N-[6-(hydroxy)cyclohex-3-enyl]carbamate [(1S,6S)-11]. Prepared by tert-butoxycarbonylation of $(1 S, 6 S)-\mathbf{1 0}$ following the method described for $(1 S, 2 S)-8$. The title compound was isolated as a white solid (99\%); mp $95-96^{\circ} \mathrm{C} ; R_{f}$ (hexane/AcOEt 3:1) 0.28; $[\alpha]_{\mathrm{D}}^{20}+30.5$ (c 1.0, $\left.\mathrm{CHCl}_{3}\right)$, ee 95\%; $\nu_{\max }\left(\mathrm{CH}_{2} \mathrm{Cl}_{2}\right) 3428$, 2980, 1693, 1503, $1171 \mathrm{~cm}^{-1} ; \delta_{\mathrm{H}}\left(300 \mathrm{MHz} \mathrm{CDC1}_{3}\right)$ 5.62-5.50 (m, $2 \mathrm{H}, \mathrm{CH}=\mathrm{CH}), 4.66(\mathrm{br} \mathrm{s}, 1 \mathrm{H}, \mathrm{NH}), 3.78-3.58(\mathrm{~m}, 2 \mathrm{H}, \mathrm{H}-1+\mathrm{H}-6), 2.92$ (br s, $1 \mathrm{H}, \mathrm{OH}), 2.57-2.43(\mathrm{~m}, 2 \mathrm{H}), 2.18-2.00(\mathrm{~m}, 1 \mathrm{H}), 2.00-1.90(\mathrm{~m}$, $1 \mathrm{H}), 1.45\left(\mathrm{~s}, 9 \mathrm{H},{ }^{t} \mathrm{Bu}\right) ; \delta_{\mathrm{C}}\left(75.5 \mathrm{MHz} \mathrm{CDC}_{3}\right) 156.7(\mathrm{C}=\mathrm{O}), 124.6(\mathrm{CH}=$ $\mathrm{CH}), 124.2(\mathrm{CH}=\mathrm{CH}), 79.7(\mathrm{C}), 70.1(\mathrm{CH}), 52.1(\mathrm{CH}), 33.5\left(\mathrm{CH}_{2}\right), 31.3$ $\left(\mathrm{CH}_{2}\right), 28.2\left(\mathrm{CH}_{3}\right) ; \mathrm{m} / z\left(\mathrm{ESI}^{+}\right) 236\left(100, \mathrm{MNa}^{+}\right) ; \mathrm{HRMS}\left(\mathrm{ESI}^{+}\right): \mathrm{MNa}^{+}$, found 236.1266. $\mathrm{C}_{11} \mathrm{H}_{19} \mathrm{NNaO}_{3}$ requires 236.1257.

4.2.9. tert-Butyl (1S,2S)-N-(2-hydroxycyclohexyl)carbamate [(1S,2S)12]. To a mixture of $(1 S, 6 S)-11(27 \mathrm{mg}, 0.13 \mathrm{mmol})$ and $10 \% \mathrm{Pd}-\mathrm{C}$ $(13 \mathrm{mg}$ ) under a hydrogen atmosphere, methanol $(1.0 \mathrm{~mL})$ was added. The mixture was stirred under the hydrogen atmosphere at room temperature for $10 \mathrm{~h}$. After this time, the catalyst was filtered through a pad of Celite ${ }^{\circledR}$ and washed with methanol. The filtrate was concentrated to reduced pressure to give pure the title compound (1S,2S)-12 (99\%). Spectroscopic data are in good agreement with those previously reported. ${ }^{15}[\alpha]_{\mathrm{D}}^{20}+22.6($ c $0.50, \mathrm{MeOH})$, ee 95\%. Ref. 15: $[\alpha]_{\mathrm{D}}^{20}+19.19($ c $0.50, \mathrm{MeOH})$, enantiopure.

4.2.10. tert-Butyl (1S,6R)-N-[6-(azido)cyclohex-3-enyl]carbamate [(1S,6R)-13]. It was obtained by previous mesylation of $(1 S, 6 S)-\mathbf{1 1}$ $(0.115 \mathrm{~g}, 0.54 \mathrm{mmol})$ with mesyl chloride following the procedure described. ${ }^{18}$ Then, the crude mesyl derivative was dissolved in anhydrous $N, N$-dimethylformamide $(1.0 \mathrm{~mL})$ and sodium azide $(0.105 \mathrm{~g}, 1.62 \mathrm{mmol})$ was added. After heating the mixture at $70^{\circ} \mathrm{C}$ during $24 \mathrm{~h}$, ethyl acetate $(5 \mathrm{~mL})$ was added and the organic solution was repeatedly washed with water. The organic phase was dried with $\mathrm{Na}_{2} \mathrm{SO}_{4}$, and evaporated to reduce pressure to give 
a residue, which was subjected to flash chromatography (hexane/ AcOEt 30:1; hexane/AcOEt 20:1; dichloromethane were successively used as eluent). ${ }^{19}$ The title compound (1S,6R)-13 was obtained as a white solid (63\%); mp $72-73{ }^{\circ} \mathrm{C} ; R_{f}$ (hexane/AcOEt 3:1) $0.51 ;[\alpha]_{\mathrm{D}}^{20}-60.1\left(\right.$ c $\left.1.0, \mathrm{CHCl}_{3}\right)$, ee $=95 \% ; \nu_{\max }\left(\mathrm{CH}_{2} \mathrm{Cl}_{2}\right) 2980$, $2130,1634,1340,1160 \mathrm{~cm}^{-1} ; \delta_{\mathrm{H}}\left(300 \mathrm{MHz} \mathrm{CDC1}_{3}\right) 5.70-5.50(\mathrm{~m}$, $2 \mathrm{H}, \mathrm{CH}=\mathrm{CH}) ; 4.72(\mathrm{br} \mathrm{s}, 1 \mathrm{H}, \mathrm{NH}), 4.00-3.82(\mathrm{~m}, 2 \mathrm{H}, \mathrm{H}-1+\mathrm{H}-6)$, $2.58-2.40(\mathrm{~m}, 1 \mathrm{H}), 2.38-2.29(\mathrm{~m}, 2 \mathrm{H}), 2.11-2.01(\mathrm{~m}, 1 \mathrm{H}), 1.44(\mathrm{~s}$, $\left.9 \mathrm{H},{ }^{t} \mathrm{Bu}\right) ; \delta_{\mathrm{C}}\left(75.5 \mathrm{MHz} \mathrm{CDC}_{3}\right)$ 155.1 $(\mathrm{C}=\mathrm{O}), 125.1(\mathrm{CH}=\mathrm{CH}), 122.6$ $(\mathrm{CH}=\mathrm{CH}), \quad 79.6(\mathrm{C}), \quad 59.1(\mathrm{CH}), 48.1(\mathrm{CH}), 29.0\left(\mathrm{CH}_{2}\right), 28.3$ $\left(\mathrm{CH}_{3}+\mathrm{CH}_{2}\right) ; \mathrm{m} / \mathrm{z}\left(\mathrm{ESI}^{+}\right) 261\left(100, \mathrm{MNa}^{+}\right)$; HRMS $\left(\mathrm{ESI}^{+}\right): \mathrm{MNa}^{+}$, found 261.1318. $\mathrm{C}_{11} \mathrm{H}_{18} \mathrm{~N}_{4} \mathrm{NaO}_{2}$ requires 261.1322 .

4.2.11. (3aS,7aR)-3a,4,7,7a-Tetrahydrobenzo[d]oxazol-2(3H)-one [(3aS,7aR)-14]. It was isolated after the flash chromatography of the residue obtained in the previous reaction with $27 \%$ yield. White solid; mp 53-55 ${ }^{\circ} \mathrm{C} ; R_{f}$ (AcOEt) 0.37; $[\alpha]_{\mathrm{D}}^{20}+49.7$ (c 1.0, $\mathrm{CHCl}_{3}$ ), ee 95\%; $\nu_{\max }\left(\mathrm{CH}_{2} \mathrm{Cl}_{2}\right) 2973,1684,1634,1167 \mathrm{~cm}^{-1} ; \delta_{\mathrm{H}}(300 \mathrm{MHz}$ $\left.\mathrm{CDC1}_{3}\right) 6.12$ (br s, $\left.1 \mathrm{H}, \mathrm{NH}\right), 5.91$ (br s, $\left.2 \mathrm{H}, \mathrm{CH}=\mathrm{CH}\right), 4.98-4.84(\mathrm{~m}$, $1 \mathrm{H}, \mathrm{O}-\mathrm{CH}), 4.16-4.04(\mathrm{~m}, 1 \mathrm{H}, \mathrm{N}-\mathrm{CH}), 2.58-2.44(\mathrm{~m}, 1 \mathrm{H}, \mathrm{CHH})$, 2.24-2.02 (m, 3H, $\left.\mathrm{CH}_{2}+\mathrm{CHH}\right)$; $\delta_{\mathrm{C}}\left(75.5 \mathrm{MHz} \mathrm{CDC1}_{3}\right) 160.1(\mathrm{C}=\mathrm{O})$, $126.6(\mathrm{CH}=\mathrm{CH}), 125.9(\mathrm{CH}=\mathrm{CH}), 75.3(\mathrm{CH}), 50.4(\mathrm{CH}), 28.4\left(\mathrm{CH}_{2}\right)$, $27.6\left(\mathrm{CH}_{2}\right) ; \mathrm{m} / z\left(\mathrm{ESI}^{+}\right) 162\left(100, \mathrm{MNa}^{+}\right), 140\left(60, \mathrm{MH}^{+}\right)$; HRMS $\left(\mathrm{ESI}^{+}\right)$: $\mathrm{MH}^{+}$, found $140.0706 . \mathrm{C}_{7} \mathrm{H}_{10} \mathrm{NO}_{2}$ requires 140.0704 .

4.2.12. (1S,2R)-N-(tert-Butoxycarbonyl)cyclohex-4-ene-1,2-diamine $[(1 S, 2 R)-15]$. It was prepared by Staudinger reduction of azidocarbamate $(1 S, 6 R)$-13 following a previously described procedure. ${ }^{18}$ Flash chromatography (AcOEt/methanol 10:1 as eluent) of the crude of the reaction yielded compound $(1 S, 6 R)-\mathbf{1 5}(68 \%)$ as a colourless oil; $R_{f}(\mathrm{AcOEt} / \mathrm{MeOH} 20: 1) 0.17 ;[\alpha]_{\mathrm{D}}^{20}-14.7\left(c 1.0, \mathrm{CHCl}_{3}\right)$, ee 95\%; $\nu_{\max }\left(\mathrm{CH}_{2} \mathrm{Cl}_{2}\right) 3355,3281,2976,1692,1535,1167 \mathrm{~cm}^{-1} ; \delta_{\mathrm{H}}$ $\left(300 \mathrm{MHz} \mathrm{CDC1}_{3}\right) 5.52(\mathrm{~s}, 2 \mathrm{H}, \mathrm{CH}=\mathrm{CH}), 5.00(\mathrm{br} \mathrm{s}, 1 \mathrm{H}, \mathrm{NH}), 3.75(\mathrm{br}$ $\mathrm{s}, 1 \mathrm{H}, \mathrm{H}-1), 3.10-3.01(\mathrm{~m}, 1 \mathrm{H}, \mathrm{H}-2), 2.47-2.25(\mathrm{~m}, 2 \mathrm{H}), 2.06-1.82$ $(\mathrm{m}, 2 \mathrm{H}), 1.62-1.38$ [br s+s, $11 \mathrm{H} ; \mathrm{NH}_{2}$ (br s, $\left.1.53 \mathrm{ppm}\right)$ and ${ }^{t} \mathrm{Bu}(\mathrm{s}$, $1.42 \mathrm{ppm})$ ]; $\delta_{\mathrm{C}}\left(75.5 \mathrm{MHz} \mathrm{CDC1}_{3}\right) 155.8(\mathrm{C}=\mathrm{O}), 124.4(\mathrm{CH}=\mathrm{CH}), 79.1$ (C), $49.4(\mathrm{CH}), 48.2(\mathrm{CH}), 33.0\left(\mathrm{CH}_{2}\right), 29.1\left(\mathrm{CH}_{2}\right), 28.3\left(\mathrm{CH}_{3}\right) ; \mathrm{m} / \mathrm{z}$ $\left(\mathrm{ESI}^{+}\right) 213\left(100, \mathrm{MH}^{+}\right)$; HRMS $\left(\mathrm{ESI}^{+}\right)$: $\mathrm{MH}^{+}$, found 213.1592. $\mathrm{C}_{11} \mathrm{H}_{21} \mathrm{~N}_{2} \mathrm{O}_{2}$ requires 213.1598 .

\subsection{HPLC determination of the enantiomeric excesses}

The ee for each compound obtained in the enzymatic resolutions as well as for the cis-diamine derivative $\mathbf{1 5}$ was determined by chiral HPLC using Chiralpack-IA column. Compounds $\mathbf{5}$ and $\mathbf{7}$ were analyzed directly. Amino ester $\mathbf{9}$ was previously converted into the amino alcohol 5 by hydrolysis with a $0.50 \mathrm{M}$ methanolic solution of $\mathrm{NaOH}$. trans-Diamine 6 was transformed into its tert-butyl carbamate derivative $\mathbf{8}$. The chemoenzymatically prepared cis-amino carbamate 15 was treated with acetic anhydride and the resulting amido carbamate analysed. Results obtained in the analysis of racemic samples are as follows:

Amino alcohol $( \pm)-\mathbf{5}$ : hexane/ethanol 98:2, $0.8 \mathrm{~mL} / \mathrm{min}, 30{ }^{\circ} \mathrm{C}$; $t_{\mathrm{R}}=8.9(1 R, 6 R)$ and $11.6(1 S, 6 S) \mathrm{min} ; R_{\mathrm{S}}=4.3$.

Amino amide $( \pm)-7$ : hexane/ethanol 95:5, $0.8 \mathrm{~mL} / \mathrm{min}, 30{ }^{\circ} \mathrm{C}$; $t_{\mathrm{R}}=10.4(1 R, 2 R)$ and $14.4(1 S, 2 S) \mathrm{min} ; R_{\mathrm{S}}=6.0$.
Amino carbamate $( \pm)-8$ : hexane/ethanol $98: 2,0.8 \mathrm{~mL} / \mathrm{min}$, $30{ }^{\circ} \mathrm{C} ; t_{\mathrm{R}}=5.6(1 R, 2 R)$ and $6.8(1 S, 2 S) \mathrm{min} ; R_{\mathrm{S}}=3.3$.

Amido carbamate derived from $( \pm)-15$ : hexane/propan-2-ol 90:10, $0.8 \mathrm{~mL} / \mathrm{min}, 30{ }^{\circ} \mathrm{C} ; t_{\mathrm{R}}=17.3(1 S, 2 R)$ and $20.4(1 R, 2 S) \mathrm{min}$; $R_{\mathrm{S}}=2.6$.

\section{Acknowledgements}

Financial support from the Spanish MICINN (CTQ2007-61126) is gratefully acknowledged. F.J.Q. thanks the Spanish MICINN for a predoctoral fellowship.

\section{References and notes}

1. Kim, C. U.; Lew, W.; Williams, M. A.; Liu, H.; Zhang, L.; Swaminathan, S. Bischofberger, N.; Chen, M. S.; Mendel, D. B.; Tai, C. Y.; Laver, W. G.; Stevens, R. C. J. Am. Chem. Soc. 1997, 119, 681-690.

2. (a) For a review, see: Magano, J. Chem. Rev, 2009, 109, 4398-4438; (b) Werner, L.; Machara, A.; Sullivan, B.; Carrera, I.; Moser, M.; Adams, D. R.; Hudlicky, T.; Andraos, J. J. Org. Chem. 2011, 76, 10050-10067 and references therein cited; (c) Kim, H.-K.; Park, K.-J. J. Tetrahedron Lett. 2012, 53, 1561-1563.

3. Fukuta, Y.; Mita, T.; Fukuda, N.; Kanai, M.; Shibasaki, M. J. Am. Chem. Soc. 2006 $128,6312-6313$.

4. Mita, T.; Fukuda, N.; Roca, F. X.; Kanai, M.; Shibasaki, M. Org. Lett. 2007, 9 , 259-262.

5. (a) González-Sabín, J.; Gotor, V.; Rebolledo, F. Chem.-Eur. J. 2004, 10, 5788-5794; (b) González-Sabín, J: Gotor, V; Rebolledo, F. J. Org. Chem. 2007 72, 1309-1314; (c) Quijada, F. J.; González-Sabín, J.; Rebolledo, F.; Gotor, V. Tetrahedron 2009, 65, 8028-8034.

6. For some reviews about vicinal amino alcohols, see: (a) Bergmeier, S. C. Tetrahedron 2000, 56, 2561-2576; (b) Lee, H.-S.; Kang, S. H. Synlett 2004 1673-1685; (c) Della Sala, G.; Russo, A.; Lattanzi, A. Curr. Org. Chem. 2011, 15 $2147-2183$

7. For some reviews about vicinal diamines, see: (a) Bennani, Y. L.; Hanessian, S Chem. Rev. 1997, 97, 3161-3196; (b) Lucet, D.; Le Gall, T.; Mioskowski, C. Angew. Chem., Int. Ed. 1998, 37, 2580-2627; (c) Kotti, S. R. S. S.; Timmons, C.; Li, G. Chem. Biol. Drug Des. 2006, 67, 101-114; (d) González-Sabín, J.; Rebolledo, F.; Gotor, V. Chem. Soc. Rev. 2009, 38, 1916-1925; (e) Alfonso, I. Curr. Org. Synth. 2010, 7 , $1-23$.

8. Perlman, N.; Albeck, A. Synth. Commun. 2000, 30, 4443-4449.

9. (a) Chen, C. S.; Fujimoto, Y.; Girdaukas, G.; Sih, C. J. Am. Chem. Soc. 1982, 104 7294-7299; (b) Program 'Selectivity' http://borgc185.kfunigraz.ac.at/sites/research/model.htm.

10. This combination of acyl donor and solvent had a beneficial effect in the CAL-Bcatalyzed resolution of some racemic amines: González-Sabín, J.; Gotor, V.; Rebolledo, F. Tetrahedron: Asymmetry 2004, 15, 481-488.

11. For some examples using PSL-C as catalyst in the resolution and desymmetryzation of amines, see: (a) Torre, O.; Busto, E.; Gotor-Fernández, V.; Gotor, V. Adv. Synth. Catal. 2007, 349, 1481-1488; (b) Ríos-Lombardía, N.; Busto, E. Gotor-Fernández, V.; Gotor, V. J. Org. Chem. 2011, 76, 5709-5718.

12. Kazlauskas, R. J.; Weissfloch, A. N. E.; Rappaport, A. T.; Cuccia, L. A. J. Org. Chem 1991, 56, 2656-2665.

13. Garro-Helion, F.; Merzouk, A.; Guibé, F. J. Org. Chem. 1993, 58, 6109-6613.

14. Thus far, lipase from Burkholderia cepacia (before classified as Pseudomonas cepacia) has proven to be the most effective catalyst for the resolution of secondary alcohols. See for example: Bornscheuer, U. T.; Kazlauskas, R. J. Hydrolases in Organic Synthesis Regio- and Stereoselective Biotransformations, 2nd ed.; Wiley-VCH: Weinheim, 2006, Chapter 5.

15. Okano, M.; Mito, J.; Maruyama, Y.; Masuda, H.; Niwa, T.; Nakagawa, S.-i.; Nakamura, Y.; Matsuura, A. Bioorg. Med. Chem. 2009, 17, 119-132.

16. Benedetti, F; Norbedo, S. Tetrahedron Lett. 2000, 41, 10071-10074.

17. Peña, C.; González-Sabín, J.; Rebolledo, F.; Gotor, V. Tetrahedron: Asymmetry 2008, 19, 751-755.

18. Quijada, F. J.; Gotor, V.; Rebolledo, F. Org. Lett. 2010, 12, 3602-3605.

19. Beside the compound $(1 S, 6 R)-\mathbf{1 3}$, the bicyclic oxazolidinone 14 was also isolated in this reaction. Characterization of $\mathbf{1 4}$ is given in section 4.2.10. 